\title{
Machine and Mechanism Design at UMinho: Research-Teaching Interaction
}

\author{
M. Lima, E. Seabra and L. F. Silva \\ University of Minho, Portugal, e-mail: \{mlima, eseaba, lffsilva\}@dem.uminho.pt
}

\begin{abstract}
The Multiweave project involved the design of a prototype machine with several mechanisms, namely gear trains and various conjugate cams with oscillating roller followers. For this design phase the research team integrated postgraduate student work. In order to evaluate the quality of the manufactured cam profiles as well as the accuracy of the follower's displacement, an experimental work has been designed. This study, which was carried out within a final year Mechanical Engineering student's group project, was of great importance in the development of the prototype machine to produce multidirectional woven fabrics, which was the main objective of the Multiweave project. The whole set-up is presented with the experimental details and the results are analysed and discussed. This measurement system is based on a laser triangulation sensor Micro-Epsilon ILD 1302-50 to measure with no contact and high resolution. The obtained results were compared with the expected theoretical values and it could be concluded that, in general, the experimental results adjust very well to the theoretical ones. However it was possible to conclude about the existence of some differences due to clearances in joints, geometric differences in the kinematic chain and manufacturing defects on the follower in terms of irregularities and surface roughness as well as some manufacturing imperfections of the cam surface.
\end{abstract}

Key words: Multiweave, multiaxial fabric, cam mechanisms, laser measurements

\section{Introduction}

In the Multiweave project a prototype weaving machine to produce a multidirectional woven fabric was investigated, designed and manufactured [1,2, and 3]. This is an innovative textile structure designed to compensate the usual anisotropic properties of conventional biaxial fabrics, being of great importance for technical applications such as in the reinforcement of special composite materials requiring improved mechanical properties for high technology applications. Fig. 1 is an example of such fabrics made from high tenacity polyester and carbon fibres and the Multiweave prototype is partially shown in Fig. 2. The concept of the machine contains several mechanisms such as for the movements of the reed, heddle and weft insertion, all in perfect synchronization and achieved by means of conjugate cam mechanisms. 


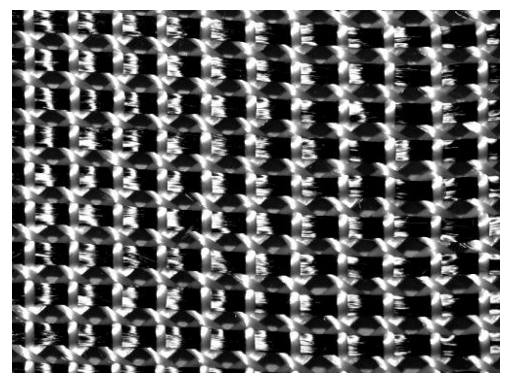

Fig. 1 Multiweave fabric sample

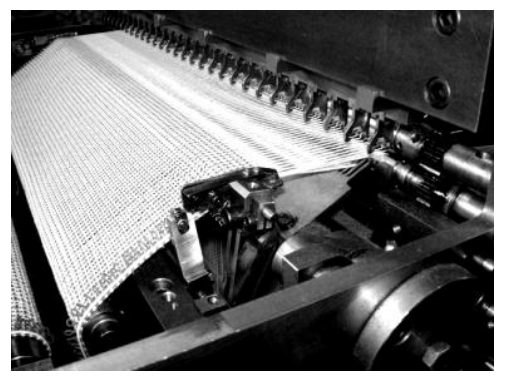

Fig. 2 Multiweave prototype

\section{Cams design and manufacturing}

The proper design of cam mechanisms is usually one of the most important and difficult steps in machine design. In the case of Multiweave and following the practice of the industry, conjugate cams were recommended [4]. Spring return is not acceptable for two main reasons: it would considerably increase the already high torque required in the cam shaft and, in the case of failure to follow the cam profile, it would result in unacceptable collisions with catastrophic results. The work was therefore concerned with the process of designing and manufacturing such mechanisms. A model of a conjugate cam with oscillating roller follower is shown in Fig. 3 where the basic design parameters are represented, namely,

$\mathrm{D}_{\mathrm{b}}$ - Cam base diameter

$\mathrm{D}_{\mathrm{r}}-$ Roller diameter

c - Distance from cam axis to follower pivot

1 - Follower arms length

$\beta$ - Follower arms angle

$\mathrm{xb}, \mathrm{yb}$ - Coordinates of follower pivot from cam axis

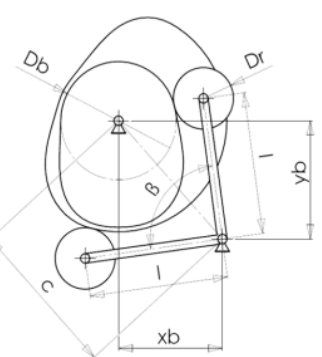

Fig. 3 Conjugate cams geometric model 
For the modelling a program was created in MATLAB $\AA$ environment which is capable of computing single or conjugate cams with oscillating or translating roller followers and contains equations for 10 different methods. In our case, modified sine method has been used [4]. Furthermore it allows inputting an unlimited number of sections for rising, dwell and return. The final part, after completing the design with the help of CAD software is obtained in a CNC machine.

As an example, the conjugate cams of the weft insertion mechanism were obtained as represented in Fig. 4. The complete 3D SolidWorks ${ }^{\circledR}$ design of one of the two conjugate cams is represented in Fig. 5, after adding several elements such as a large centre hole for reduced mass and 4 fixing holes.

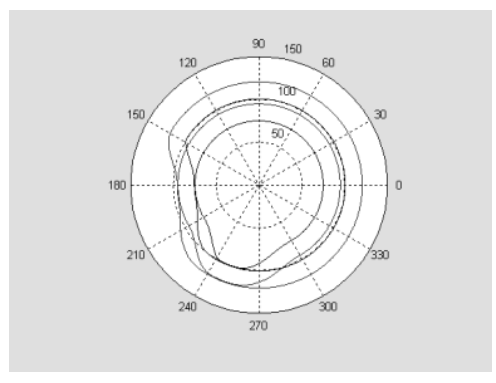

Fig. 4 Cam profiles and pitch curves as obtained from MATLAB

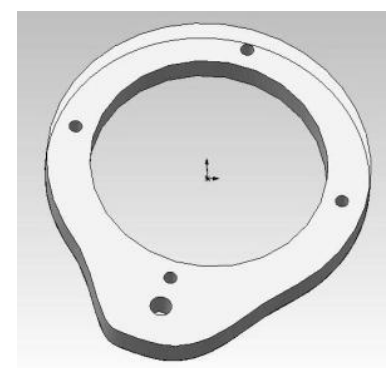

Fig. 5 3D design of one cam

One of the cam boxes of the prototype, with two sets of conjugate cams can be seen in Fig. 6, partially immersed in the lubrication oil. Also clearly visible are the swinging followers with two arms and the respective contact rollers.

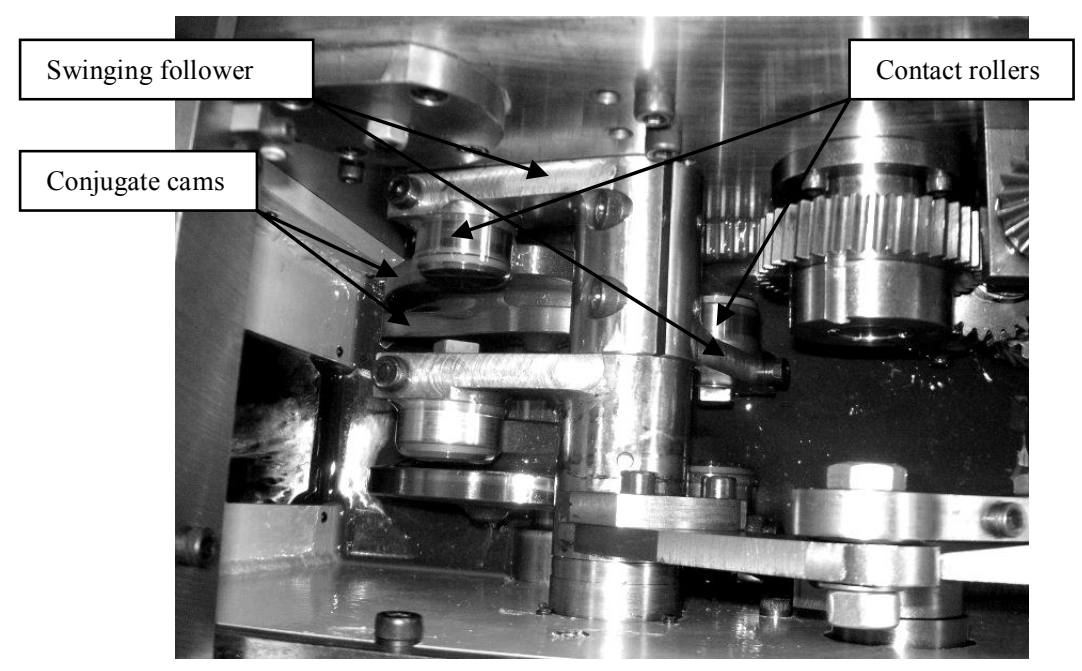

Fig. 6 Conjugate cams in cam box 
The mechanism of the weft insertion can be seen in Fig. 7 a) and b), where conjugate cams with oscillating roller follower move a four bar linkage to drive a gear train to act upon the final pinion and flexible rack mechanism.
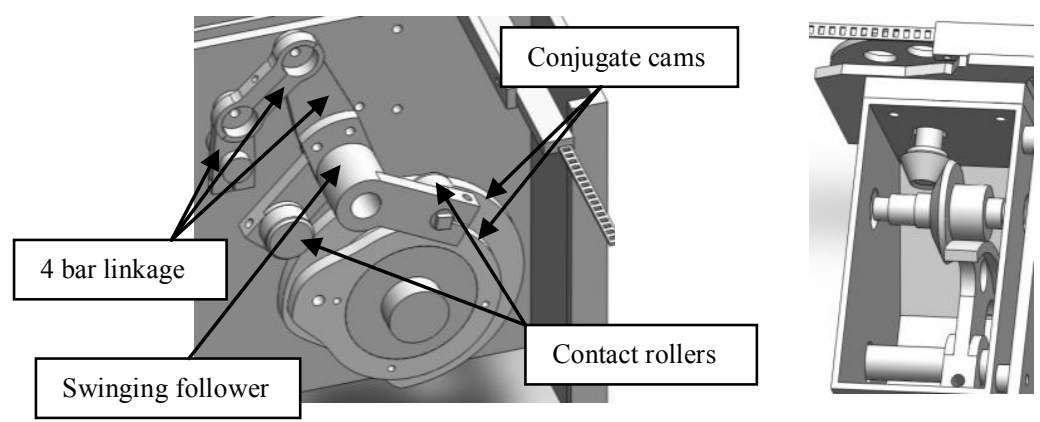

a) Conjugate cams and 4 bar linkage

b) Transmission to flexible rack

Fig. 7 Details of the weft insertion mechanism

\section{Students project work}

Part of a final year students project, an experimental work was planned and carried out in order to analyse the kinematics of the weft insertion cam mechanisms in situ and to confirm the predictions of the theoretical model as well as to verify any deviations, possibly due to manufacturing errors, assembling problems and play in joints. This work involved the development of a measurement system based on a laser triangulation sensor to measure, with no contact and high resolution, the followers' oscillating displacements of the conjugate cams represented in the cam box of Fig. 6. A laser sensor Micro-Epsilon ILD 1302-50 [5, 6], with a resolution of $10 \mu \mathrm{m}$ has been used involving two measurements: the follower displacement and the cams profiles. Very low speed was used in order to minimise vibrations. Fig. 8 represents the geometry when measuring the follower position $(A=143,7$ $\mathrm{mm} ; \mathrm{B}=92,4 \mathrm{~mm}, \mathrm{C}=43,1^{\circ}$ ) and Fig. 9 when measuring the cams profiles $\left(\mathrm{A}=234,3 \mathrm{~mm} ; \mathrm{B}=37,9 \mathrm{~mm}, \mathrm{C}=163,1^{\circ}\right)$.

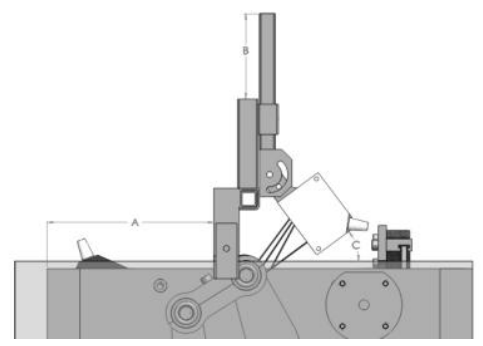

Fig. 8 Measurement of follower position

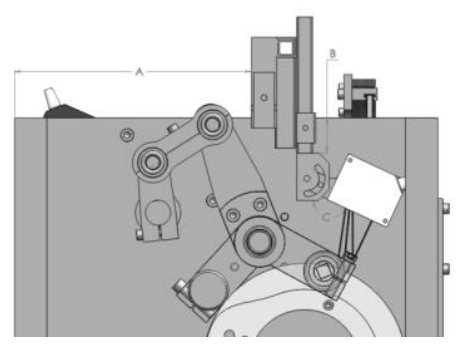

Fig. 9 Measurement of cams profiles 
To accomplish this experimental work, a PC-based commercial data acquisition hardware has been used together with a software specifically developed for this purpose using the LabVIEW® platform from National Instruments.

\section{Results and discussion}

Fig. 10 represents the experimental data versus the theoretical values for the cam follower displacement, as obtained with the set-up shown in Fig. 8. It can be concluded that, in general, the experimental results follow the theoretical values for the modified sine equation. However, looking at the results with more detail, there are some visible differences between the experimental and theoretical values, as shown in figure 11.

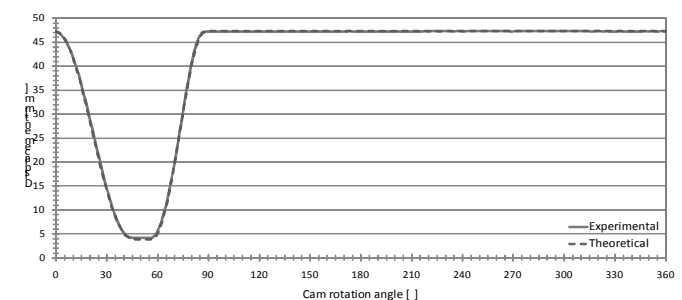

Fig. 10 Experimental data versus theoretical values for the cam follower displacement

These visible differences in the follower movements (rise and return), happened, approximately, between $0^{\circ}$ and $90^{\circ}$, which can be linked to measuring errors due to the curvature of the follower's surface and consequently, perpendicularity loss between the laser beam and the measured surface, existence of clearances in joints and differences between designed and real cam profiles, originating a different follower displacement.

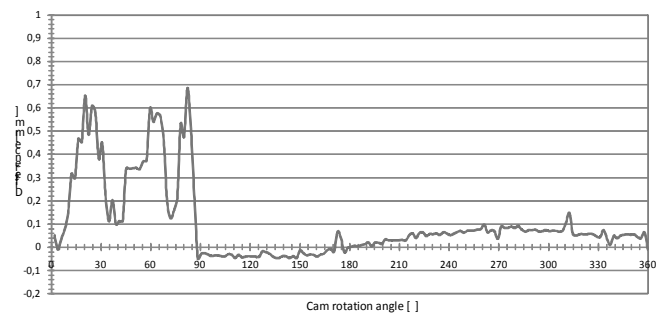

Fig. 11 Differences between experimental and theoretical values for the cam follower displacement of figure 10

It can also be observed that, approximately between $0^{\circ}$ and $90^{\circ}$ the experimental data are greater than the expected theoretical values, which may be due to exis- 
tent clearances in joints and geometric differences in the kinematic chain, besides the ones existing in the follower shaft.

On the other hand, it is also visible the occurrence of a peak in the beginning of the follower's return movement, approximately between $55^{\circ}$ and $60^{\circ}$, that can be related once again to existent clearances in joints, manufacturing defects on the follower surface (in terms of irregularities and surface roughness), and to other manufacturing imperfections of the cam surface.

Finally, between $90^{\circ}$ and $360^{\circ}$, it can be also observed two types of problems: the first one, which is related to a sinusoidal variation of the differences (approximately with the same width), can be induced by the noise, surface roughness, measuring interferences; and, the second one, due to a quick ascent followed by a descent movement (curve with two concavities, first negative and then positive), which was probably created by a cam off-centred or by a manufacturing defect.

Fig. 12 and 13 respectively present the experimental data versus the theoretical values for the rise and return displacement of the cam profiles, as obtained by the set-up shown in Fig. 9. Globally, it can be concluded that, in both cases, the experimental results follow the theoretical values. However, as it is highlighted in detail in Figs. 14 and 15, there are some observable differences between the experimental and theoretical values.

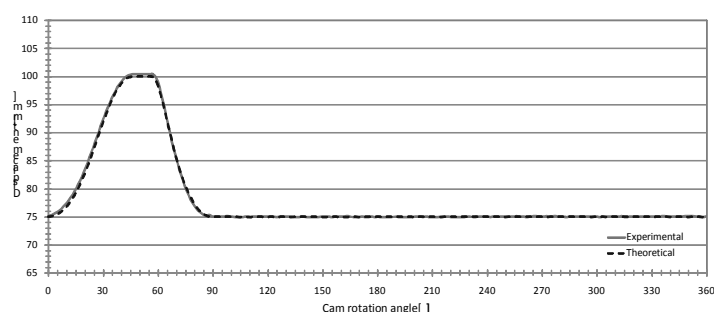

Fig. 12 Experimental data versus theoretical values for the rising cam profile

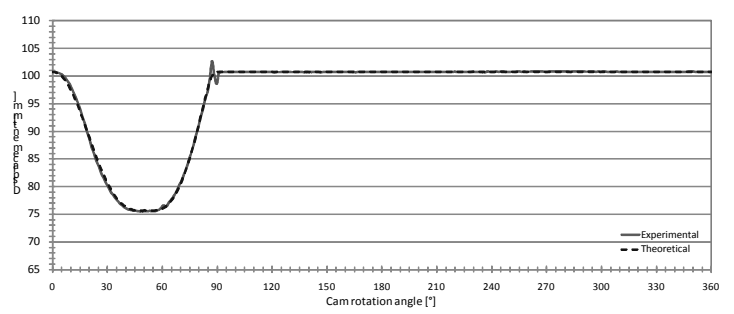

Fig. 13 Experimental data versus theoretical values for the return cam profile

These observable differences in the cams profiles (rise and return), occurred, approximately, between the rotation angles of $0^{\circ}$ and $90^{\circ}$. This could have been derived from the measuring errors due to the cams curvatures, cams off-centred, and designed cams profiles different from the real cams profiles, originating different follower's displacements. 
It can also be observed in the rising cam, approximately between $45^{\circ}$ and $55^{\circ}$, that the experimental data are greater than the expected theoretical values, while for the return cam the opposite occurs. As in previous cases, it can be due to cams off-centred and manufacture defects in the cams profiles.

It is also possible to observe the clear occurrence of two peaks: one in the rising cam at the beginning of the follower's return movement (around $60^{\circ}$ ), and another in the return cam at the ending of the follower's rising movement (around $87^{\circ}$ ). Once again these peaks can be related to some manufacturing defects on the cams surfaces, where it is possible to confirm (at naked eye) local irregularities.

Finally, it can also be observed, between the rotation angles of $90^{\circ}$ and $360^{\circ}$, two types of problems, which, for the authors, are of the same nature of those described in the analysis carried out for the cam follower displacement.

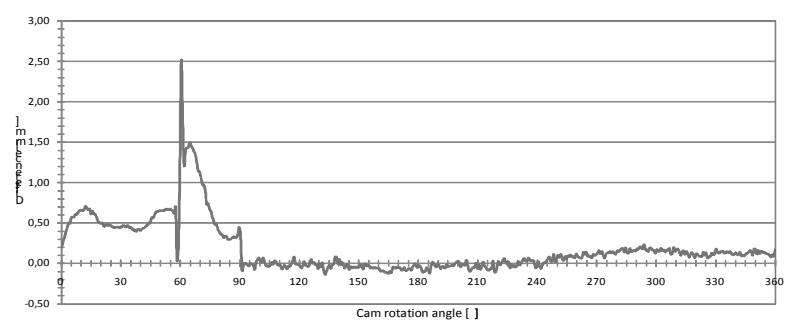

Fig. 14 Differences between experimental and theoretical values for the rising cam profile

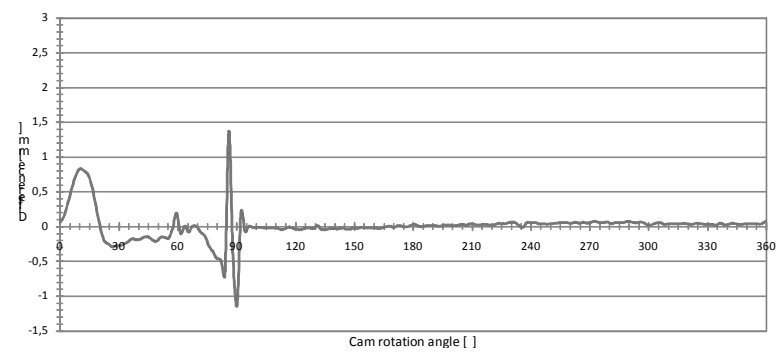

Fig. 15 Differences between experimental and theoretical values for the return cam profile

Due to the main errors identified in the follower movement and in the cams profiles, and their consistency (occurring in the same angular positions), it is possible to conclude that the acquisition system designed and developed specifically for this purpose (based on a laser triangulation sensor) proved to be very appropriate to measure, with no contact and with high resolution, the follower oscillating movements (rise and return) of conjugate cams. On the other hand, based on the angles of the detected cam position errors, it allowed the evaluation of the errors magnitude in the cams and follower. According to the computed results, it was also possible to conclude that the magnitude of the obtained differences between the theoretical values and experimental data are similar for the follower and cams. 


\section{Conclusions}

The interaction between teaching and research has been achieved with success. In the design phase the research team integrated postgraduate student work. Later an experimental study was carried out within a final year Mechanical Engineering student's group project to evaluate the conformity of various mechanisms.

As a result, conjugate cams could be obtained using a MATLAB ${ }^{\circledR}$ based program which allowed a very fast design process. The designer can quickly obtain and compare several different designs, and, on the basis of the analysis of the kinematics characteristics, optimize the mechanism. The result can easily be exported to a $\mathrm{CAD}$ tool to create the complete $3 \mathrm{D}$ design of the cams. The final $\mathrm{CAD}$ file is directly used as a source data for the CNC manufacturing.

An investigation was carried out to evaluate the quality of the manufactured cam profiles as well as the accuracy of the follower displacement. An experimental work was designed and carried out based on a measurement system using a laser triangulation sensor Micro-Epsilon ILD 1302-50 to measure with no contact and high resolution. The obtained results were compared with the expected theoretical values. It could be concluded that, in general, the experimental readings follow the theoretical values but with some visible differences between the measured results and the expected theoretical values which could be explained by the clearances in joints, geometric differences in the kinematic chain and manufacturing defects on the follower surface in terms of irregularities and surface roughness as well as some manufacturing imperfections of the cam surface. This study has been of great importance to improve the quality of the manufactured mechanisms and to the development of the Multiweave prototype.

\section{References}

[1] Lima, M., et al. "MULTIWEAVE - Multiaxial Weaving: From Concept to Prototype", AUTEX 2007 International Conference, 7th Annual Textile Conference by Autex, From Emerging Innovations to Global Business, 26-28 June 2007, Tampere, Finland, ISBN 978952-15-1794-5.

[2] Lima, M., et al. "MULTIWEAVE - Prototype Weaving Machine for Multiaxial Technical Fabrics", ALTEX 2007, 20-22nd September 2007, Roubaix, France. In ALTEX 2007 Conference proceedings, CD ROM.

[3] Lima, M., et al. "MULTIWEAVE - Prototype Weaving Machine for Multiaxial Technical Fabrics", Indian Journal of Fibre \& Textile Research, Vol. 34, March 2009, pp 59-63.

[4] Lima, Mário, Zabka, Petr. "Design and Analysis of Conjugate Cam Mechanisms for a Special Weaving Machine Application", 2nd International Conference on Innovations, Recent Trends and Challenges in Mechatronics, Mechanical Engineering and New High-Tech Products Development - MECAHITECH'10, Bucharest, Romania, 23-24 Sep, 2010.

[5] Micro-Epsilon, "ILD 1302", http://www.micro-epsilon.com/index.html, as visited on 2011.07.29.

[6] Micro-Epsilon, “optoNCDT-1302”, http://www.cdiweb.com/datasheets/micro_epsilon/dax-optoNCDT-1302--en.pdf, as visited on 2011.07.29. 